\title{
A Real-time Rectification using an Adaptive Differential Encoding for High-resolution Video
}

\author{
Jong-hak Kim*, Jung-kyun Oh**, Seong-muk Kang*** and Jun-dong Cho*** \\ *Department of IT convergence, Sungkyunkwan University, Korea \\ **Department of Human ICT convergence, Sungkyunkwan University, Korea \\ ***Department of Electrical and Computer Engineering, Sungkyunkwan University, Korea \\ ihakkim@vada.skku.ac.kr, ikoh@vada.skku.ac.kr, smkang@vada.skku.ac.kr, jdcho@skku.edu
}

\begin{abstract}
Previously, various rectification methods using compressed lookup table have been studied for real-time hardware stereo vision system. These loss compression methods may occur distortion that could corrupt disparity estimation process. Differentially encoded lookup table method which is lossless compression has no distortion with reasonable compression ratio. However, the method is limited to lowresolution and low warping movement. In this paper, we propose an adaptively differential encoding method. Our proposed algorithm has approximately $18 \sim 26 \%$ compression ratio which is comparable to the previous method and tolerates high-resolution video.
\end{abstract}

Keywords - real-time processing, high-resolution rectification, lookup-table, adaptive differential encoding, lossless compression

\section{INTRODUCTION}

A stereo vision system has four steps. The first step is rectification as a pre-processing. A role of rectification is to align epipolar lines. And the second step is disparity estimation to obtain disparity-map. In the third step, filtering techniques are utilized to enhance disparity-map. Finally, disparity-map is used for various applications such as visual fatigue decrease, object segmentation and tracking, motion control and etc. Since rectification not only aligns epipolar lines but also decreases search-range for disparity estimation process, it is essential process to achieve real-time processing. Figure 1 shows reduced search-range.

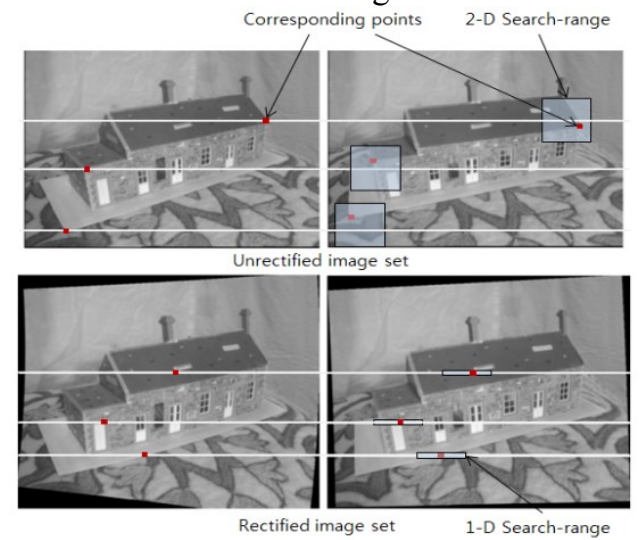

Figure 1. Example of reduced search-range[1]
Rectification could categorize into real-time calculation and pre-calculation using a LUT (Lookup table). Since real-time calculation consist a large amount of computation, many research tried to simplify calculation of epipolar geometry matrix as in [2]-[4]. And a hardware architecture using FPGA (Field-programmable gate array) is also used to achieve realtime processing as in [5]-[7]. However, simplified calculation methods are not suitable for real-time processing. And FPGA methods could process in real-time, but they consist of complex hardware architecture.

On the other hand, pre-calculation using a LUT has fast processing-time with compact hardware architecture as in [8]. But disadvantage of this method is high memory consumption. To overcome this issue, loss and lossless compression techniques are applied. One of the loss compression methods subsamples rectification mapping data and reconstructs using interpolation as in [9], [10]. Another recent research uses the run-length encoding technique with different order as in [11]. Although the loss compression method has much higher compression ratio than the lossless compression method, it also has distortion, and could be critical for the performance of disparity estimation process. To avoid distortion, reference [12] uses differential encoding and decoding using only subtraction and accumulation, respectively that consumes only 1.3 Mbytes for a 1280 by 720 image with $27 \%$ compression ratio. However, it limits data magnitude bits to 3 , so data magnitude have to be less than 3 which is not suitable for high-resolution rectification.

In this paper, we propose an adaptive differential encoding method. We analysed probability of appearance of differential values and built a new codebook including four modes. The first mode is comprised of minimum bit size to decrease compression ratio, and the fourth mode could extent bit size for magnitude to tolerate high-resolution video.

The rest of paper is organized as follows. We will introduce basic concept of rectification using LUT in Section II. Our proposed algorithm will be presented in Section III. Proposed hardware architecture will be presented in Section IV. Experimental result will be given in Section V, and finally, we will draw the conclusion in Section VI. 


\section{BASIC CONCEPT OF RECTIFICATION USING LUT}

Rectification is used for various applications such as orthophoto or orthoimage as in [13], [14], multi-view camera system as in [6], [15], intelligent vehicle using stereo vision system as in [16], [17] and etc. Difference between stereo vision system and others is that position of each camera is fixed, which needs only one set of rectification parameters if there is no individual movement of each camera and uses it consistently. Therefore, we are allowed to pre-calculate rectification mapping data and rectify images by loading mapping data from LUT memory.

Rectification using LUT could divide into calibration and rectification procedures. The calibration procedure is to calculate rectification movement using intrinsic parameters, extrinsic parameters and camera calibration factors and obtain rectification mapping data. The mapping data includes coordinate information that moves pixels on the original image to the rectified image. This procedure processes in software environment. And the rectification procedure compensates input images using mapping data from the LUT memory. Since complex computation is mostly calculated in the calibration procedure, the rectification procedure could be designed compact hardware architecture and be suitable for real-time processing. However, there are four different mapping data set which are $\mathrm{X}$ and $\mathrm{Y}$ coordinate information from left and right images. And it affects high memory consumption. Therefore, each mapping data set ought to be compressed. Compressed LUT methods are simply added encoding and decoding parts before and after LUT memory, respectively. And complexity increase depends on which compression method is utilized. Compressed LUT methods classify loss and lossless compression. Figure 2 shows overall flow of rectification using LUT.

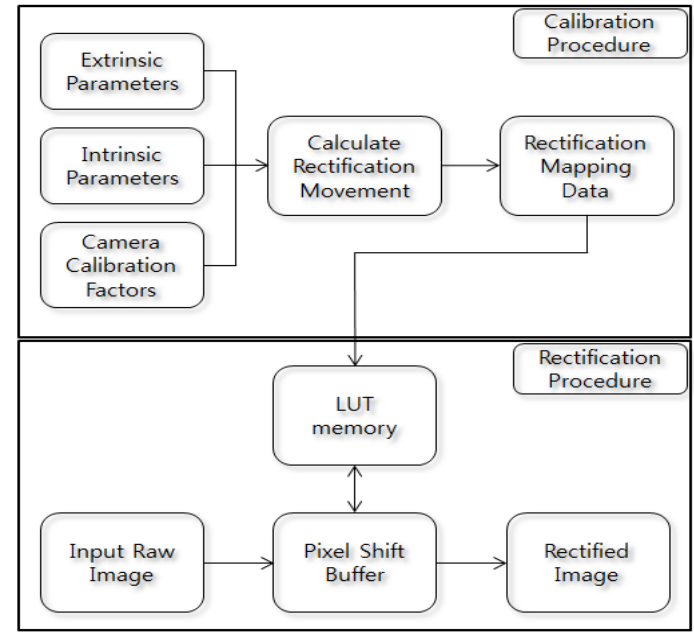

Figure 2. Overall flow of rectification using LUT

\section{A. Loss compression}

The loss compressed LUT method usually applies downsampling to reduce a size of LUT in the encoding process and interpolation to fill void pixels in the decoding process as in [9], [10]. Given that interpolation change calculated values based on relationship with adjacent values, a rectified image is distorted. Although rectified image using a loss compressed LUT shows reasonable PSNR over $30 \mathrm{~dB}$, it could occur critical errors in the disparity estimation process. To avoid potential errors, the lossless compression is more suitable.

\section{B. Lossless compression}

The lossless compressed LUT method utilizes lossless compression technique such as Huffman, differential, runlength and etc. Among them, a differential encoding method is used with high compression ratio and compact hardware architecture as in [12]. It shows good trade-off between complexity and compression ratio. However, since it limits differential magnitude of sequential pixel values on 3 to minimize memory consumption, an error is occurred on the current coordinate if magnitude of differential value is more than 4. Moreover, when one error is occurred, it is propagated on the current line by accumulation of erroneous difference. Therefore, the previous differential encoding method operates properly only with low-resolution or low warping movement.
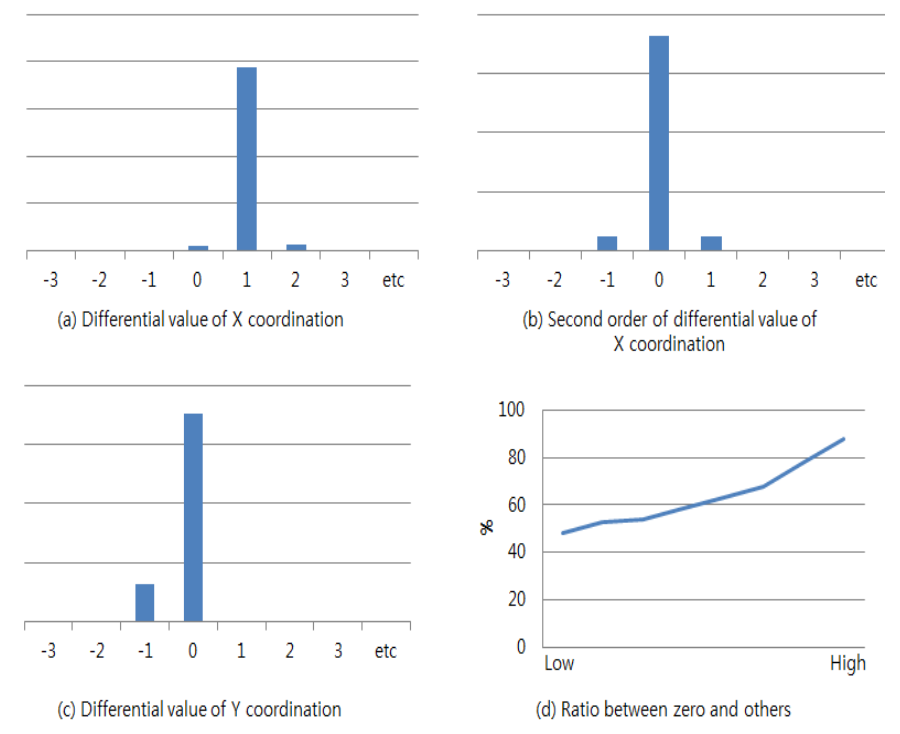

Figure 3. Distribution of difference value (Top-left) differential X coordinate value, (Top-right) the second order differential $\mathrm{X}$ coordinate value (Bottom-left) differential Y coordinate value, (Bottom-right) ratio between the highest probability case and others.

\section{Proposed ALgorithm}

Adaptive compression method basically uses characteristic of input. Reference [18] adjusted sampling frequency depend on frequency of ECG (electrocardiogram) signal. Especially, ECG signal with low frequency sampled with low sampling frequency, which decrease amount of data. In the same manner, our proposed method adjusted size of memory bits to store coordinate data depend on magnitude of differential values. Size of memory bits is minimized to decrease compression ratio for the highest probability case and also maximized to have enough bit size to store maximum magnitude for the opposite case. Therefore, compression ratio is decreased with increasing the highest probability case. 
As shown in figure 3(a) and 3(c), variance of differential coordinate values is distributed around 1,0 for $\mathrm{X}$ and $\mathrm{Y}$ coordinate, respectively. And the second order differential $\mathrm{X}$ coordinate values are distributed around 0 in figure 3(b). A value of the highest probability case in figure $3(\mathrm{~b})$ is large in comparison with the one in figure 3(a). And distribution is almost same as in figure 3(b) so that we could share the same codebook to encode $\mathrm{X}$ and $\mathrm{Y}$ coordinate values. Moreover, as shown in figure $3(\mathrm{~d})$, since the ratio between the highest probability case and others increases with higher resolution, our proposed method is more suitable for higher resolution. To satisfy above-mentioned conditions, we composed a new codebook as shown in table 1.

TABLE 1. A CODEBOOK FOR ADAPTIVE DIFFERENTIAL ENCODING

\begin{tabular}{|c|c|c|c|c|}
\hline $\begin{array}{c}\text { Range of } \\
\text { magnitude }\end{array}$ & 0 & $\pm 1 \sim \pm 2$ & $\pm 3 \sim \pm 6$ & $\pm 7 \sim$ \\
\hline Mode bit & 00 & 01 & 10 & 11 \\
\hline Sign bit & - & 1 bit & 1 bit & 1 bit \\
\hline Magnitude bit & - & 1 bit & 2 bits & 3 bits $\sim$ \\
\hline Total bits & 2 bits & 4 bits & 5 bits & 6 bits $\sim$ \\
\hline
\end{tabular}

The difference value that has the highest probability case sets as mode ' 00 ' to decrease compression ratio. And to cover larger range of magnitude, the codebook could rearrange by expansion of magnitude bit in mode ' 11 '. Let's assume that we have differential value 0 and -5 . First, 0 is included in mode ' 00 '. And mode ' 00 ' has no sign bit and magnitude bit. Therefore, 0 is encoded as ' 00 '. Second, -5 is included in mode ' 10 '. Sign is minus and magnitude is 5 . We can assign mode bit as '10', sign bit as ' 1 ', magnitude bit as ' 10 '. Therefore, -5 is encoded as ' 10110 '. The encoding flow is shown in figure 4.

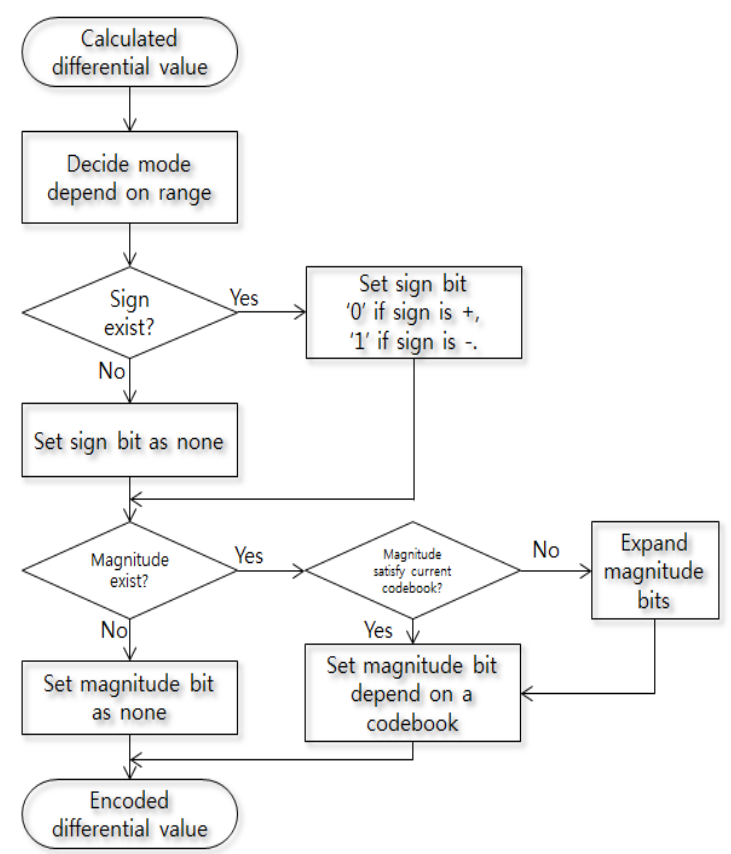

Figure 4. Encoding flow

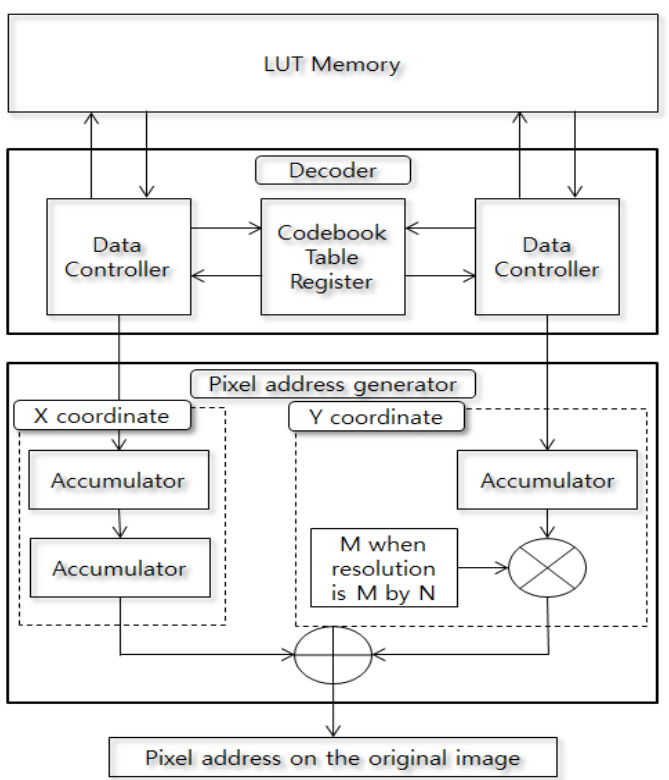

Figure 5. Proposed hardware architecture

\section{Proposed HARDWARE ARCHITECTURE}

Our proposed hardware architecture consists of LUT memory, a decoder and a pixel address generator. First, the decoder reads differentially encoded data from LUT memory and decode into $\mathrm{X}$ and $\mathrm{Y}$ coordinate values. Then the pixel address generator reconstruct $\mathrm{X}$ and $\mathrm{Y}$ coordinate values into pixel address on the original image. The decoder consists of data controller and codebook table registers and the pixel address generator consists of two accumulators for $\mathrm{X}$ coordinate, one accumulators and one multiplier for $\mathrm{Y}$ coordinate and one adder to combine $\mathrm{X}$ and $\mathrm{Y}$ coordinate values. Overall hardware architecture is compact with low latency and simple computation, which is suitable for realtime processing. The proposed hardware architect is shown in figure 5 .

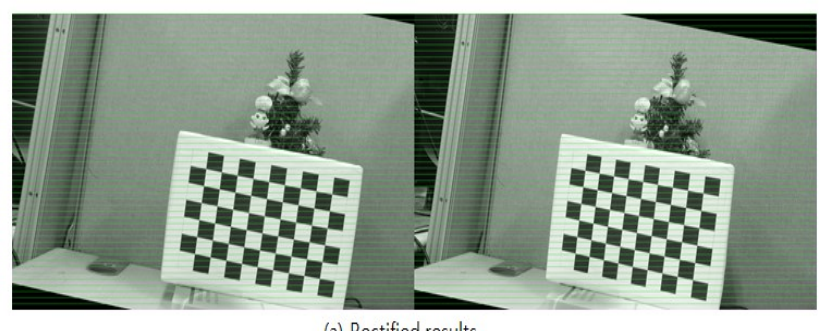

(a) Rectified results

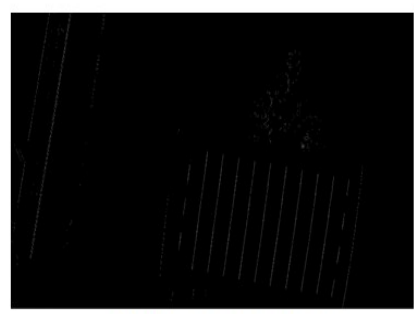

(b) Differential binary image using abstract encoding[9]

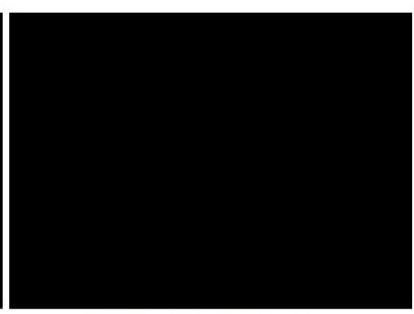

(c) Differential binary image using proposed encoding
Figure 6. Differential binary image 


\section{EXPERIMENTAL RESULT}

Our proposed method is designed using OpenCV for the encoding process and Verilog for the decoding process. For the evaluation, we applied two methods. The first one is a binary image of difference to show distortion. The equation for binarization is as:

$$
\mathrm{B}(\mathrm{x}, \mathrm{y})=\left\{\begin{array}{l}
0 \text { if Recti_img } \\
1 \text { if Recti_img } \text { if }_{(x, y)} \neq \text { Ori_img }_{(x, y)}, \\
\text { Ori_img } \\
(x, y)
\end{array}\right. \text {. }
$$

Since distortion is the same as difference, distortion regions represent with white pixels in the result images. As shown in figure 7, the result images of the loss compression method have white regions, whereas our proposed method does not

The second method is compression ratio. We compared to previous differential encoding method as in [12] with various resolution conditions. Compression ratio is defined as:

$$
\mathrm{CR}=\frac{\text { Compressed data }}{\text { UnCompressed data }}
$$

As shown in figure 8 , compression ratio of our proposed method has minimum $18.9 \%$ and maximum $26.1 \%$ lower than the previous one. And difference of compression ratio in the same resolution becomes larger with higher resolution.

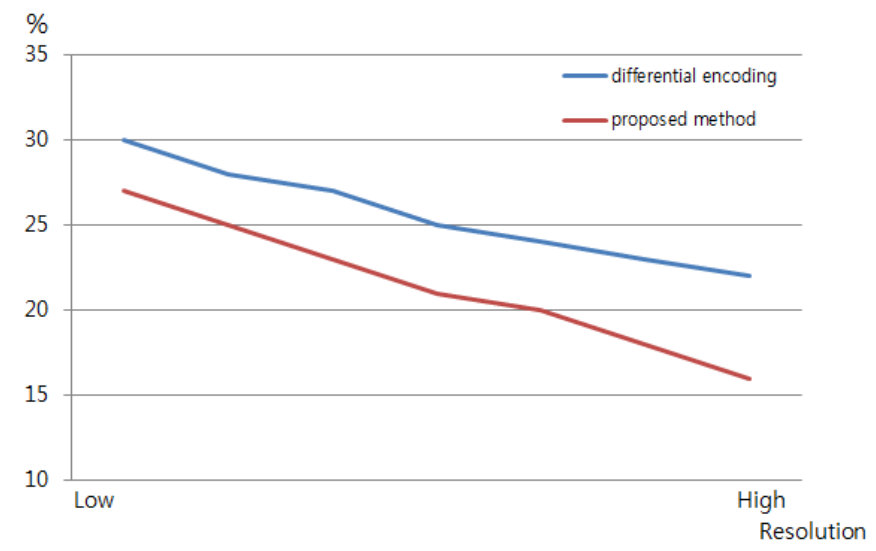

Figure 7. Compression ratio results with various resolutions

\section{VI.CONCLUSIONS}

In this paper, we proposed an adaptive differential encoding method for high-resolution video. Our proposed method effectively encodes satisfying two conditions such as minimization to decrease compression ratio and maximization to tolerate large magnitude values. And decoding process also achieves compact hardware architecture with processing in real-time. Compression ratio is approximately from $18.9 \%$ to $26.1 \%$ depending on resolution with no distortion. And the higher resolution shows the lower compression ratio which is suitable for image processing with high-resolution.

\section{ACKNOWLEDGMENT}

Following are results of a study on the "Leaders in Industry-university Cooperation" Project, supported by the Ministry of Education, Science \& Technology (MEST).

This research was supported by Basic Science Research Program through the National Research Foundation of Korea(NRF) funded by the Ministry of Education(NRF2013R1A1A2058942)

\section{REFERENCES}

[1] Yunseok Lee, Kar-Ann Toh and Sangyoun Lee, "Stereo image rectification based on polar transformation," Optical Engineering, 47(8), 087205, 2008.

[2] Nicholas Ayache and Charles Hansen, "Rectification of Images for Binocular and Trinocular Stereovision," in Proc. Pattern Recognition, vol. 1, pp. 11-16, Nov, 1988.

[3] Marc Pollefeys, Reinhard Koch and Luc Van Gool, "A Simple and Efficient Rectification method for General Motion," in Proc. Computer vision, vol. 1, pp 496-501, Sep, 1999.

[4] Andrea Fusiello, Emanuele Trucco and Alessandro Verri, "A Compact Algorithm for Rectification of Stereo pairs," Machine Vision and Applications, Vol. 12, pp. 16-22, 2000.

[5] S. Jin, J. Cho, X. D. Pham, K. M. Lee, S. K. Park, M. Kim and J. W. Jeon, "FPGA Design and Implementation of a Real-time Stereo Vision System," IEEE Trans. Circuits and Systems for Video technology, Vol. 20, No. 1, pp. 15-26, Jan, 2010.

[6] Minsu Choi, Jinsang Kim, Won-Kyung Cho and Yunmo Chung, "Low Complexity Image Rectification for Multi-view Video Coding," in Proc. ISCAS 2012, pp. 381-384, May, 2012.

[7] Heiko Hubert, Benno Stabernack and Frederik Zilly, "Architecture of a Low Latency Image Rectification Engine for Stereoscopic 3-D HDTV Processing," IEEE Trans. Circuits and Systems for Video technology, Vol. 23, No. 5, pp. 813-822, May, 2013.

[8] Cristian Vancea and Sergiu Nedevschi, "LUT-based Image Rectification Module Implemented in FPGA," in Proc. Intelligent Computer Communication and Processing, pp. 147-154, Sep, 2007.

[9] Anders Kjaer-Nielsen, Lars Baunegaard With Jensen, Anders Stengaard Sorensen and Norbert Kruger, "A Real-time Embedded System for Stereo Vision Preprocessing using an.FPGA," in Proc. Reconfigurable Computing and FPGAs, pp. 37-42, Dec, 2008.

[10] Khurram Jawed, John Morris, Tariq Khan and Georgy Gimel'farb, "Real time Rectification for Stereo Correspondence," in Proc. Computational Science and Engineering, pp. 277-284, Aug, 2009.

[11] Abdulkadir Akin, Ipek Baz, Luis Manuel Gaemperle, Alexandre Schmid and Yusuf Leblebici, "Compressed Look-Up-Table based Real-Time Rectification Hardware," in Proc. VLSI-SoC 2013, pp. 272 277, Oct, 2013.

[12] Deuk Hyun Park, Hyoung Seok Ko, Jae Gon Kim and Jun Dong Cho, "Real Time Rectification using Differentially Encoded Lookup Table," in Proc. ICUIMC 2011, No. 47, Feb, 2011.

[13] Ayman F. Habib, Eui-Myoung Kim and Chang-Jae Kim, "New Methodologies for True Orthophoto Generation," Photogrammetric Engineering \& Remote Sensing, Vol. 73, No. 1, pp. 25-36, Jan, 2007.

[14] W. Karel, M. Doneus, G. Verhoeven, C. Briese, C. Ressl, N. Pfeifer, "Oriental - Automatic Geo-referencing and Ortho-rectification of Archaeological Aerial Photographs," ISPRS Annals of the Photogrammetry, Remote Sensing and Spatial Information Science, Vol II-5/W1, 2013, XXIV International CIPA Symposium, Sep, 2013.

[15] Yun-Suk Kang, Cheon Lee and Yo-Sung Ho, "An Efficient Rectification Algorithm for Multi-view Images in Parallel Camera Array," in Proc. 3DTV conference: The True Vision- Capture, Transmission and Display of 3D Video, pp. 61-64, May, 2008.

[16] Wannes van der Mark and Dariu M. Gavrila, "Real-time Dense Stereo for Intelligent Vehicles," IEEE Trans. Intelligent transportation systems, Vol. 7, No. 1, March, 2006.

[17] Anwar Hasni Abu Hasan, Rostam Affendi Hamzah and Mohd Haffiz Johar, "Range Estimation in Disparity Mapping for Navigation of Stereo Vision Autonomous Vehicle using Curve Fitting Tool," IJVIPNS, Vol. 9, No. 9, pp. 5 9, Oct, 2009.

[18] Zhongyun Yuan, Jong-hak Kim and Jun-dong Cho, "Adaptive Sampling for ECG Detection based on Compression Dictionary," JSTS, in press.

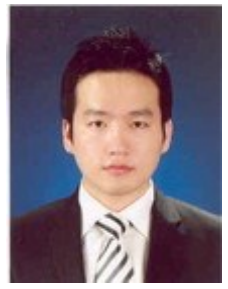

Jong-Hak Kim received the B.S. degree in radio communication engineering from the Kyunghee University, Suwon, Korea, in 2009, the M.S. degree from the Department of Electrical and Computer Engineering, Sungkyunkwan University, in 2012, and he is studying for a $\mathrm{Ph}$. D degree at Sungkyunkwan University. He is interested in the efficient low power 
hardware implementation for a real-time image processing system in mobile equipment. He currently studies a visual fatigue reduction scheme for a stereo vision system.

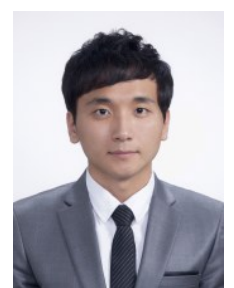

Jung-kyun Oh received the B.S degree in Information Communication Engineering from Myongji University, Yongin, Korea, in 2013. He is a M.S candidate in the

Human ICT Convergence Department at

Sungkyunkwan University, Suwon, Korea.

His research interests include SoC for 3D Image

Processing Applications and 3D visual fatigue

reduction.

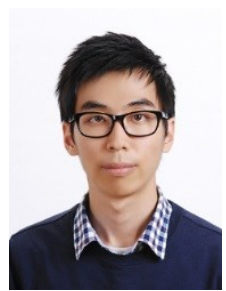

Seong-muk Kang received the B.S degree from the Department information communication from

Baekseok University, Korea, in 2013. And he is

studying for a master's degree in Electrical Engineering at Sungkyunkwan University, Suwon, Korea.

His current research interests include image

stabilization and stereo vision.

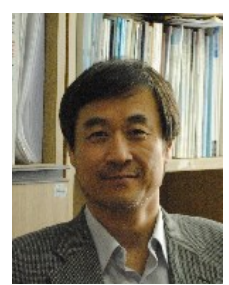

Jun-Dong Cho received the B.S. degree from the Department of Electronic Engineering, Sungkyunkwan University, Suwon, Korea, in 1980, the M.S. degree from the Department of Computer Science, Polytechnic University Brooklyn, New York, in 1989, and the Ph.D. degree from the Department of Computer Science, Northwestern University, Evanston, in 1993.

He was working for Samsung Electronics Company for 6 years. He joined the Department of Electrical and Computer Engineering, Sungkyunkwan University (SKKU), Suwon, Korea, since 1995, where he is currently chair of graduate school of Human ICT Convergence.

His research interests include Low Power Design, 3-D Image Processor, Embedded Multimedia, and Human ICT Application.

Prof. Cho is an IEEE Senior Member. 